Ophthalmologe 2019 $116: 1177-1183$ https://doi.org/10.1007/s00347-019-00998-2 Online publiziert: 27. November 2019 (c) Der/die Autor(en) 2019

Jan Tjeerd de Faber ${ }^{1}$ Tero T. Kivelä ${ }^{2} \cdot$ Ameli Gabel-Pfisterer $^{3}$

${ }^{1}$ Augenklinik Rotterdam, Rotterdam, Niederlande

${ }^{2}$ Klinik für Augenheilkunde, Universität und Universitätsklinikum Helsinki, Helsinki, Finnland

${ }^{3}$ Augenklinik, Klinikum Ernst-von-Bergmann Potsdam, Potsdam, Deutschland

\title{
Landesweite Studien aus den Niederlanden und Finnland zur Häufigkeit von Augenverletzun- gen durch Feuerwerkskörper unter dem Einfluss verschiedener Schutzmaßnahmen
}

heitsempfehlungen oder gesetzliche Regulationen zum Absatz oder Gebrauch von Feuerwerkskörpern beeinflusst werden.

Augenverletzungen im Zusammenhang mit privatem Feuerwerk stellen ein ernsthaftes globales Problem dar. Die Hälfte der Verletzten sind Kinder, und ein Zehntel der Verletzten erblindet dauerhaft [5]. Länder mit restriktiven Feuerwerksvorschriften melden fast $90 \%$ weniger Augenverletzungen als Länder ohne solche Vorschriften. Der Präsident der European Society of Ophthalmology (SOE) hat einen weltweiten Aktionsplan zur Prävention von Augenverletzungen durch Feuerwerkskörper initiiert, der unter anderem auch vom International Council of Ophthalmology (ICO), der American Academy of Ophthalmology $(\mathrm{AAO})$ und der International Agency for Prevention of Blindness (IAPB) unterstützt wird. Die SOE hat ein Online-Register für feuerwerksbedingte
Augenverletzungen entwickelt, um Daten zu sammeln. Die Niederländische Gesellschaft für Augenheilkunde (NOG) mahnt strengere Regelungen im privaten Umgang mit Feuerwerkskörpern an und empfiehlt Einzelpersonen den Gebrauch von Schutzbrillen [2-4, 6]. Die Augenklinik der Universität Helsinki arbeitet seit 2 Jahrzehnten eng mit der finnischen Augenärztlichen Gesellschaft und der Finnischen Agentur für Sicherheit und Chemikalien zusammen. Sie organisiert nationale Datenerhebungen, hat sich erfolgreich für ein gezieltes Verbot der gefährlichsten Feuerwerkskörper eingesetzt und weitere Gesetzesänderungen auf der Grundlage der erhobenen Daten eingefordert. Ähnliche regulatorische Prozesse sind weltweit in anderen Ländern erforderlich. In dieser Studie möchten wir den Einfluss von öffentlichen Kampagnen und Änderungen der Gesetzgebung zum Schutz der Menschen vor schmerzhaften und visusmin- diesem Beitrag beschreiben wir die Unterschiede in der Häufigkeit dieser Augenverletzungen in verschiedenen Ländern und wie sie durch Informationskampagnen, Sicher-

Die englische Version dieses Beitrags ist unter https://doi.org/10.1007/s00347-019-00996-4 zufinden.

Der Beitrag wurde übersetzt von Dr. Ameli Gabel-Pfisterer, Potsdam.

\begin{tabular}{l|l|l|l|l|l|l|l|l|l|l|l|}
$\begin{array}{l}\text { Tab. } 1 \\
\text { frage }\end{array}$ & Alter der Verletzten in den Niederlanden (Mittel und Median) in den 11 Jahren der Um- \\
\hline Jahr & $\mathbf{2 0 0 8}$ & $\mathbf{2 0 0 9}$ & $\mathbf{2 0 1 0}$ & $\mathbf{2 0 1 1}$ & $\mathbf{2 0 1 2}$ & $\mathbf{2 0 1 3}$ & $\mathbf{2 0 1 4}$ & $\mathbf{2 0 1 5}$ & $\mathbf{2 0 1 6}$ & $\mathbf{2 0 1 7}$ & $\mathbf{2 0 1 8} \mathbf{1 9}$ \\
\hline $\begin{array}{l}\text { Mittleres } \\
\begin{array}{l}\text { Alter } \\
\text { Alter Median }\end{array}\end{array}$ & 23,3 & 23,1 & 24,5 & 27,3 & 24,3 & 25 & 25,5 & 26,2 & 26,2 & 25,2 & 25,3 \\
\hline
\end{tabular}




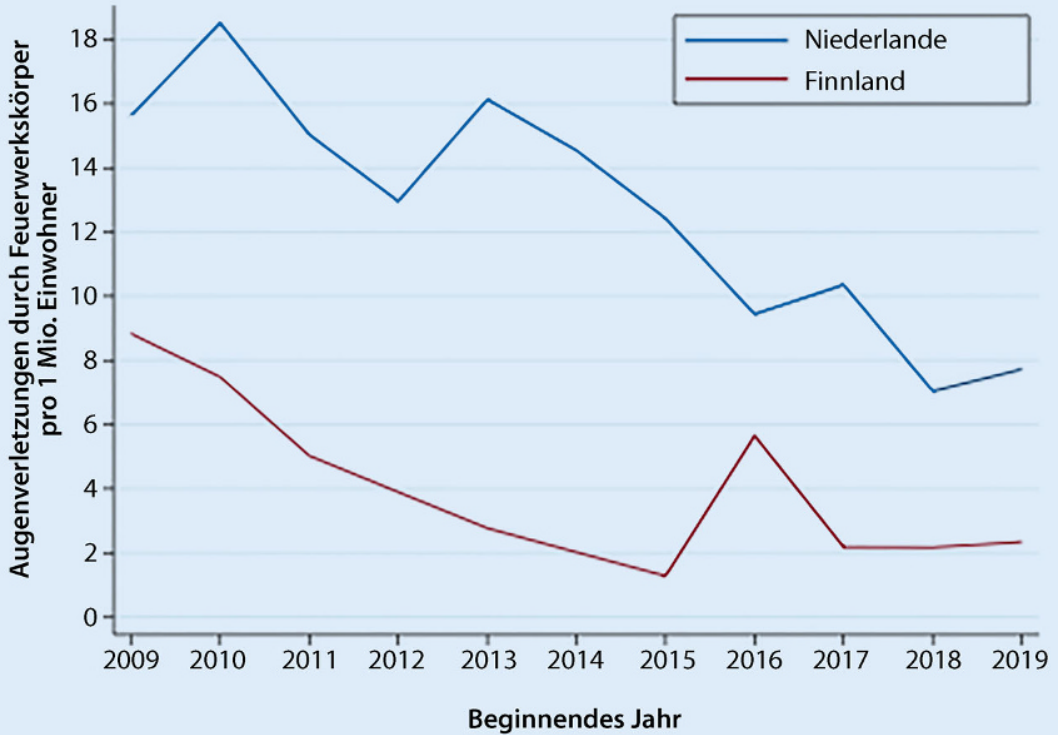

Abb. 1 A Anzahl der Augenverletzungen durch Feuerwerkskörper pro 1 Mio. Einwohner in den Niederlanden und Finnland. Der Unterschied in der Anzahl der Verletzungen pro 1 Mio. Einwohner spiegelt wahrscheinlich wider, dass die Öffentlichkeitsarbeit in Finnland fast 1 Jahrzehnt früher begann. Verschiedene Methoden der Datenerhebung können aber auch zu dem Unterschied beitragen

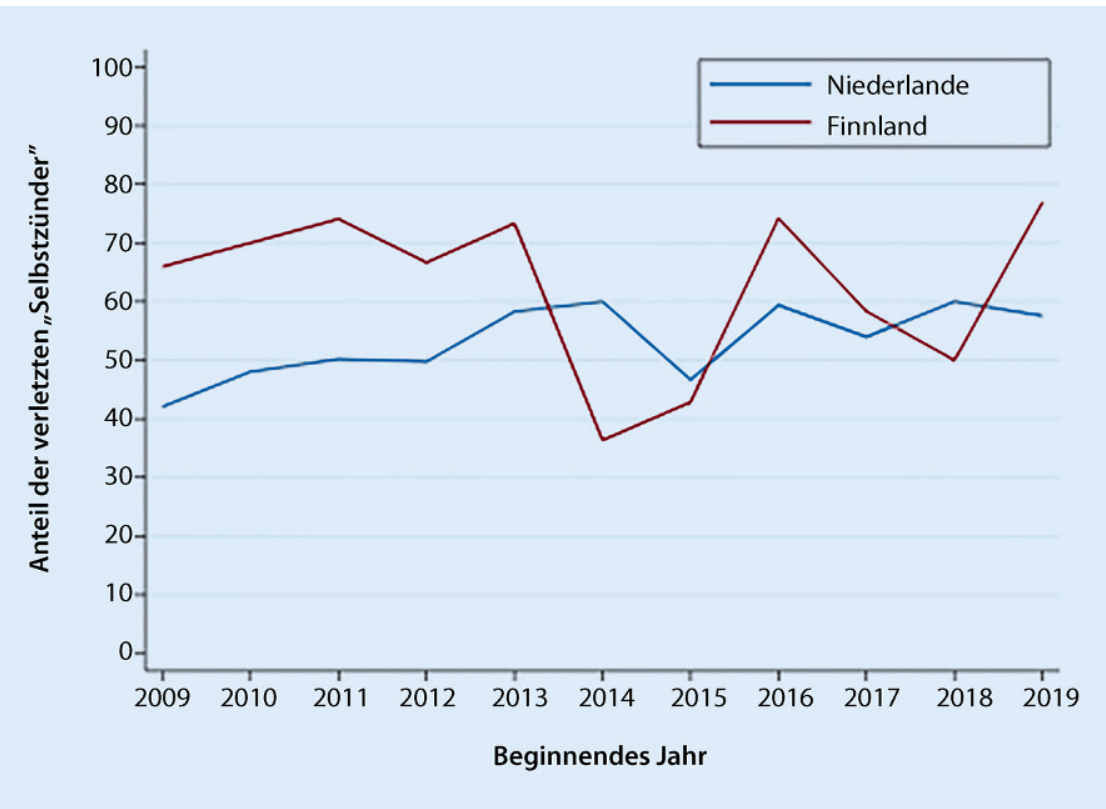

Abb. 2 A Anteil der feuerwerksbedingten Augenverletzungen bei Selbstzündern in den Niederlanden und Finnland von 2009 bis 2019

dernden Augenverletzungen durch Feuerwerkskörper untersuchen.

\section{Material und Methoden}

In den Niederlanden wird seit 11 Jahren jährlich in den Tagen um Silvester eine Erhebung von Daten von Patienten mit diesem Zeitraum gesammelt wurden, wurde eine Telefonumfrage an allen Kliniken durchgeführt, um die Teilnahmequote zu überprüfen. In den 11 Jahren der Umfrage wurde eine Teilnahmequote von $92-96 \%$ verzeichnet.

In Finnland wurde in den letzten 20 Jahren eine ähnliche Umfrage durchgeführt, die alle Krankenhäuser umfasst; sie erfasst keine Bagatellverletzungen, die von Allgemeinmedizinern ohne Rücksprache mit einem Augenarzt behandelt werden können. Die Daten aus Finnland sind hier für 2009 bis 2019 aufgeführt, um eine Vergleichbarkeit mit den niederländischen Daten zu ermöglichen. Beide Umfragen beinhalten Fragen zu Alter, Geschlecht, Unfallablauf (Zuschauer oder aktiver Feuerwerker), Art des Feuerwerks, Ausmaß der Verletzung, Therapie und angenommener Prognose. Wir korrelieren die Gesamtzahlen zeitlich mit durchgeführten öffentlichen Kampagnen und Gesetzesänderungen, um deren Auswirkungen auf die Inzidenz $\mathrm{zu}$ veranschaulichen.

\section{Ergebnisse}

\section{Alter und Geschlecht}

In den Niederlanden und in Finnland sind die meisten Verletzten junge Männer. Weniger als $10 \%$ der Verletzten sind weiblich, $50 \%$ sind Zuschauer.

Das mittlere Durchschnittsalter in den Niederlanden während der 11 Jahre der Erhebung variierte zwischen 23 und 27 Jahren und der Median des Alters zwischen 18 und 23 Jahren. Eine Verschiebung zu höheren Altersgruppen deutet auf einen besseren Schutz von Kindern hin. Das jüngste Opfer in den Niederlanden war dennoch ein 3-jähriger Zuschauer (•Tab. 1).

In Finnland lag das Durchschnittsalter über die 11 Jahre bei 25 Jahren und der Median bei 20 Jahren. Der jüngste Patient war ein 4 -jähriger Zuschauer.

\section{Feuerwerkskörper}

In den Niederlanden haben Raketen (25\%), Knallkörper (35\%) und Römische Lichter (11\%) die meisten Augenverletzungen verursacht. Ein ähnliches 
Spektrum an auslösenden Feuerwerkskörpern wurde auch in Finnland festgestellt. Aber selbst Wunderkerzen können zu schweren Augenverletzungen führen. Feuerwerksbatterien, die nur einmal gezündet werden müssen, um mehrfache Effekte auszulösen, haben in der Öffentlichkeit einen sicheren Ruf. In den Niederlanden haben sie jedoch bis $\mathrm{zu}$ $20 \%$ der schwereren Augenverletzungen mit dauerhaften Schäden am Auge verursacht.

\section{Inzidenz}

Die Inzidenz feuerwerksbedingter Augenverletzungen hat im Beobachtungszeitraum aufgrund von verstärkter Öffentlichkeitsarbeit und strengeren Vorschriften von 16 bis 18 auf 8 Verletzungen pro 1 Mio. Einwohner in den Niederlanden und von 9 auf 2 Verletzungen pro 1 Mio. Einwohner in Finnland abgenommen (• Abb. 1).

\section{Zuschauer („Bystander") und "Selbstzünder"}

Der Anteil der verletzten "Selbstzünder“, die das Feuerwerk selbst gezündet haben, lag in beiden Ländern im Beobachtungszeitraum zwischen 40 und $70 \%$, was nahelegt, dass im Mittel rund die Hälfte der Verletzten Zuschauer und Passanten sind (• Abb. 2).

\section{Sicherheitsmaßnahmen}

\section{Zeitliche Begrenzung}

In den Niederlanden wurde im Jahr 2015 die Zeit während der privates Silvesterfeuerwerk gezündet werden darf, von 16 auf 8 Stunden reduziert (siehe - Abb. 3). In Finnland trat eine vergleichbare Einschränkung bereits 2010 in $\mathrm{Kraft}$ (• Abb. 4).

\section{Schutzbrillen}

Eine nationale Kampagne zur Stärkung der Sicherheitsmaßnahmen war in den Niederlanden recht erfolgreich [3, 4]. Die Verwendung von Schutzbrillen hat insgesamt zugenommen, sie werden aber nicht immer von Selbstzündern und nur selten von Umstehenden getragen. In Finnland wird die Verwendung von Schutzbrillen

Ophthalmologe 2019·116:1177-1183 https://doi.org/10.1007/s00347-019-00998-2

(c) Der/die Autor(en) 2019

\section{J. T. de Faber · T. Kivelä · A. Gabel-Pfisterer}

\section{Landesweite Studien aus den Niederlanden und Finnland zur Häufigkeit von Augenverletzungen durch Feuerwerkskörper unter dem Einfluss verschiedener Schutzmaßnahmen}

Zusammenfassung

Feuerwerksbedingte Augenverletzungen treten weltweit v. a. bei privaten Feuerwerken auf. Zwei Register aus Finnland über 20 Jahre und aus den Niederlanden über 10 Jahre haben die Auswirkungen von Öffentlichkeitsarbeit und gesetzlichen Regelungen untersucht. Die Häufigkeit von Feuerwerksverletzungen in diesen beiden Ländern wurde in den letzten 10 Jahren auf etwa die Hälfte reduziert, indem die Anzahl der zulässigen Stunden für private Feuerwerke und strengere Vorschriften für die Verwendung von Feuerwerkskörpern festgelegt wurden. Zudem wurde die Öffentlichkeitsarbeit verstärkt und die Verwendung von Schutzbrillen empfohlen und erleichtert. Obwohl mit diesen Maßnahmen die Gesamtzahl der Verletzungen zurückgegangen ist, scheint die Schwere der Augenverletzungen in den Niederlanden möglicherweise aufgrund des höheren Ge- halts an Schwarzpulver in Feuerwerkskörpern zuzunehmen. Vor allem Jungen und junge Männer sind von Augenverletzungen durch Feuerwerkskörper betroffen, ebenso wie Zuschauer, die von mehr als der Hälfte der Verletzungen betroffen sind. Es ist noch viel zu tun, um Feuerwerke sicherer zu machen - eine Herausforderung für alle länderübergreifenden ophthalmologischen Organisationen wie European Society of Ophthalmology (SOE) und International Council of Ophthalmology (ICO) und die nationalen Gesellschaften gleichermaßen. Aber jedes Auge, das vor schweren Verletzungen bewahrt werden kann, ist die Mühe wert.

\section{Schlüsselwörter}

Privates Feuerwerk · Explosionen · Augenschutz $\cdot$ Nationale Verzeichnisse zu Augenverletzungen - Feuerwerksgesetzgebung

\section{National studies from the Netherlands and Finland and the impact of regulations on incidences of fireworks-related eye injuries. German version}

\section{Abstract}

Fireworks-related eye injuries occur all over the world whenever fireworks are available for the public. The two prospective registers from Finland over 20 years and from the Netherlands over 10 years have tracked the effect of regulations, awareness, and safety campaigns. The incidence of fireworks-related injuries in these two countries has been reduced to about one half during the last 10 years through addressing the number of hours allowed for private fireworks, the use of safety glasses, awareness campaigns, and stricter regulations on fireworks available for purchase. Although the total number of injuries has decreased, the average severity of eye injuries in the Netherlands seems to have increased due to higher content of gunpowder in fireworks. Boys and young men are mainly affected as well as bystanders who account for more than half of fireworks-related eye injuries. More work on safety of fireworks is still needed through regulating private consumer use of fireworks, a challenge that lies ahead for ophthalmologic organizations worldwide, including the European Society of Ophthalmology (SOE), the International Council of Ophthalmology (ICO) and national societies. But every eye saved from severe injury is worth the effort.

\section{Keywords}

Private fireworks - Explosions - Eye protection . National eye trauma registers . Fireworks legislation seit 1999 befürwortet, und ist seit 2010 für alle Selbstzünder gesetzlich vorgeschrieben. Jedem, der sich an Silvester im Freien bewegt, wird ebenfalls empfohlen, eine Schutzbrille zu tragen.

\section{Beschränkungen des Handels und der Auswahl von Feuerwerkskörpern}

Seit dem Jahr 2010 dürfen in Finnland Feuerwerkskörper nur noch an Volljährige abgegeben werden. Darüber hinaus 


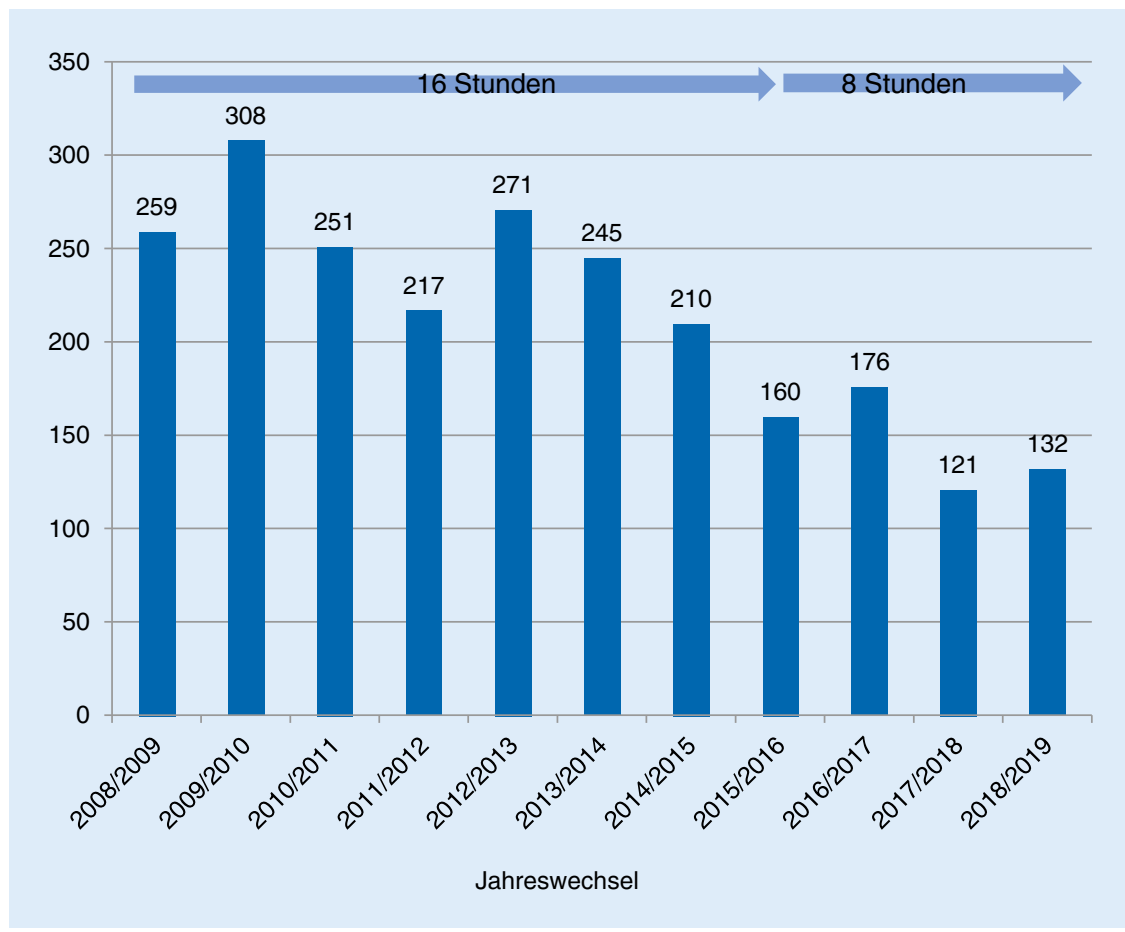

Abb. $3 \Delta$ Die Gesamtzahl der feuerwerksbedingten Augenverletzungen in den Niederlanden ist zurückgegangen, nachdem Medienkampagnen und gesetzliche Maßnahmen wie die Reduzierung der Betriebszeiten von Feuerwerkskörpern im Jahr 2015 eingeführt wurden

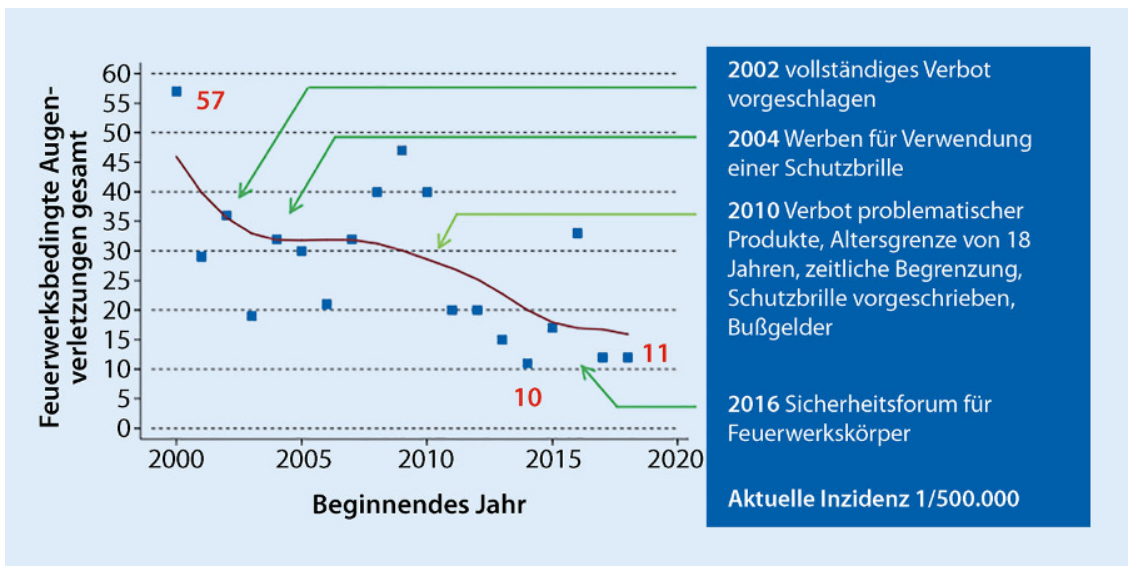

Abb. 4 \ Absolute Anzahl von Augenverletzungen in Finnland unter dem Einfluss von Öffentlichkeitsarbeit und Änderung gesetzlicher Vorgaben in den letzten 19 Jahren

wurden ab 2010 in Finnland 2 Klassen kleinerer Feuerwerkskörper, die über mehrere Jahre die meisten Augenverletzungen verursacht hatten, verboten: Römische Kerzen mit einem Durchmesser von weniger als $20 \mathrm{~mm}$ und kleine Flaschenraketen. Man kann einen deutlichen Rückgang der Anzahl der Augenverletzungen beobachten, seitdem all diese Vorschriften ihre Wirkung entfalten (•Abb. 4).
Eine weitere Schlussfolgerung der 11jährigen Umfrage in den Niederlanden ist, dass zwar weniger Augenverletzungen auftreten, der Anteil der bleibenden Augenschäden jedoch leicht zugenommen hat (•Tab.2). Dies könnte darauf zurückzuführen sein, dass seit 2014 in den Niederlanden größere Mengen Schießpulver (bis zu 200g) pro Projektil verwendet werden dürfen. Während der 11-jährigen Umfrage erlitten 2350 niederländische Verletzte 2898 verletzte Augen; 1021
Augen trugen dauerhafte Schäden davon, 190 erblindeten und 76 Augen mussten entfernt werden.

Im Gegensatz dazu hat Finnland die zulässige Menge an Schießpulver in Raketen im Jahr 2010 von $200 \mathrm{~g}$ auf $75 \mathrm{~g}$ reduziert, und der größte Durchmesser der ,aerial shells“ (Luftgranaten) wurde auf $28 \mathrm{~mm}$ begrenzt. Im gleichen Zeitraum von 11 Jahren erlitten 236 Verletzte Augenverletzungen. Davon hatten 50 Augen eine dauerhafte Visusminderung, 184 Augen erlitten leichte Verletzungen ohne bleibende Schäden. Allerdings erlitten 117 Augen Verletzungen von mittlerer Schwere, wie z. B einer verzerrten Pupille, aber ohne dauerhaften Sehverlust. Insgesamt gesehen mussten jedoch keine Augen entfernt werden. In beiden Ländern hat sich der Prozentsatz der Patienten mit Verletzungen, die einen bleibenden Schaden am Auge hinterlassen, nicht verbessert (• Abb. 5).

\section{Diskussion}

Die nationalen Register in den Niederlanden und in Finnland zeigen, dass die Inzidenz der feuerwerksbedingten Augenverletzungen in beiden Ländern unter dem Einfluss von Öffentlichkeitsarbeit und Überarbeitung der gesetzlichen Vorschriften, wie z.B. der zeitlichen Begrenzung von privatem Feuerwerk von 16 auf $8 \mathrm{~h}$ gesunken ist. Ein Verbot bestimmter Arten von Feuerwerkskörpern und Kampagnen zum Tragen von Schutzbrillen haben ebenfalls dazu beigetragen, die Zahl der Augenverletzungen in beiden Ländern zu verringern [6]. Andererseits hat in den Niederlanden offenbar der Anstieg des Sprengstoffgehalts in Feuerwerkskörpern gemäß EU-Norm die Zahl der schweren Augenverletzungen erhöht.

\section{Auswirkungen auf die Inzidenz- raten durch Vorschriften für den Einsatz von Feuerwerkskörpern und Sicherheitsmaßnahmen}

In Finnland forderte im Juni 2019 eine an das Parlament gerichtete Bürgerpetition gesetzliche Regelungen zum Verbot aller privat genutzten Feuerwerkskörper oberhalb Kategorie F1. Um angenommen $\mathrm{zu}$ werden, muss eine Petition in $6 \mathrm{Mo}-$ 


\begin{tabular}{|c|c|c|c|c|c|c|c|c|c|c|c|}
\hline Augenverletzungen & $\begin{array}{l}2008 / \\
2009\end{array}$ & $\begin{array}{l}2009 / \\
2010\end{array}$ & $\begin{array}{l}2010 / \\
2011\end{array}$ & $\begin{array}{l}2011 / \\
2012\end{array}$ & $\begin{array}{l}2012 / \\
2013\end{array}$ & $\begin{array}{l}2013 / \\
2014\end{array}$ & $\begin{array}{l}2014 / \\
2015\end{array}$ & $\begin{array}{l}2015 / \\
2016\end{array}$ & $\begin{array}{l}2016 / \\
2017\end{array}$ & $\begin{array}{l}2017 / \\
2018\end{array}$ & $\begin{array}{l}2018 / \\
2019\end{array}$ \\
\hline Patienten & 259 & 308 & 251 & 217 & 271 & 245 & 210 & 160 & 176 & 120 & 132 \\
\hline Aktiver Feuerwerker & 109 & 148 & 126 & 108 & 158 & 147 & 98 & 95 & 95 & 72 & 76 \\
\hline Umstehende & $\begin{array}{l}150 \\
(58 \%)\end{array}$ & $\begin{array}{l}160 \\
(52 \%)\end{array}$ & $\begin{array}{l}125 \\
(50 \%)\end{array}$ & $\begin{array}{l}109 \\
(50 \%)\end{array}$ & $\begin{array}{l}113 \\
(42 \%)\end{array}$ & $\begin{array}{l}98 \\
(40 \%)\end{array}$ & $\begin{array}{l}112 \\
(53 \%)\end{array}$ & $\begin{array}{l}65 \\
(41 \%)\end{array}$ & $\begin{array}{l}81 \\
(46 \%)\end{array}$ & $\begin{array}{l}48 \\
(40 \%)\end{array}$ & $\begin{array}{l}56 \\
(42 \%)\end{array}$ \\
\hline Augen & 309 & 358 & 307 & 256 & 331 & 311 & 268 & 200 & 224 & 157 & 176 \\
\hline $\begin{array}{l}\text { Vorübergehender } \\
\text { Schaden }\end{array}$ & 203 & 242 & 190 & 151 & 227 & 213 & 171 & 127 & 144 & 98 & 111 \\
\hline Dauerhafter Schaden & $\begin{array}{l}106 \\
(34 \%)\end{array}$ & $\begin{array}{l}116 \\
(32 \%)\end{array}$ & $\begin{array}{l}117 \\
(38 \%)\end{array}$ & $\begin{array}{l}105 \\
(41 \%)\end{array}$ & $\begin{array}{l}104 \\
(31 \%)\end{array}$ & $\begin{array}{l}98 \\
(32 \%)\end{array}$ & $\begin{array}{l}97 \\
(36 \%)\end{array}$ & $\begin{array}{l}73 \\
(37 \%)\end{array}$ & $\begin{array}{l}80 \\
(36 \%)\end{array}$ & $\begin{array}{l}59 \\
(38 \%)\end{array}$ & $\begin{array}{l}65 \\
(37 \%)\end{array}$ \\
\hline Visusverlust & 44 & 51 & 36 & 59 & 57 & 55 & 31 & 29 & 37 & 23 & 11 \\
\hline Erblindete Augen & 20 & 22 & 16 & 21 & 25 & 20 & 10 & 15 & 19 & 11 & 10 \\
\hline Entfernte Augen & 14 & 9 & 7 & 9 & 8 & 10 & 6 & 1 & 5 & 5 & 2 \\
\hline
\end{tabular}

naten mindestens 50.000 Unterschriften erhalten; die beantragte Gesetzesvorlage erreichte diese Zahl im Dezember 2018 bereits innerhalb von 3 Wochen.

Eine an Eltern und Kinder gerichtete nationale Kampagne, die auf die Gefahren von Feuerwerken hinweist, hat in Holland dazu beigetragen, die Zahlen von jungen Opfern zu reduzieren [3, 4]. Neben der Empfehlung, sich während des Feuerwerks in Gebäuden aufzuhalten, werden Kinder zum Tragen von Schutzbrillen motiviert, indem diese in einigen Gemeinden (z. B. in Rotterdam) kostenlos in Grund- und Mittelschulen verteilt werden.

Obwohl das Tragen von Schutzbrillen bei Feuerwerken zugenommen hat, werden sie in den Niederlanden nur von wenigen erwachsenen Selbstzündern und noch seltener von Zuschauern getragen.

In Finnland trägt die Mehrheit der Selbstzünder eine Schutzbrille, was zu einem Rückgang der schwersten Verletzungen geführt hat (•Tab.3). Da Schutzbrillen die Schwere von Augenverletzungen reduzieren können, sind penetrierende Verletzungen heute selten geworden, und das Risiko, ein Auge zu verlieren, ist in Finnland stark gesunken.

\section{Erhöhung der Zahl schwerer Augenverletzungen durch höhere Mengen an Sprengstoffen}

Eine weitere Schlussfolgerung dieser 11jährigen Untersuchung ist, dass in den Niederlanden zwar weniger Augenverletzungen auftreten, der Prozentsatz der bleibenden Schäden an den Augen jedoch zugenommen hat. Dies ist hier wohl auf den höheren Gehalt von Schwarzpulver je Projektil zurückzuführen, der sich im Rahmen der EU-Richtlinie geändert hat. So sehen wir einerseits weniger Augen mit Feuerwerksverletzungen, aber mehr Augen mit schweren Verletzungen, die wegen der erhöhten Sprengkraft mit dauerhaften Schäden rechnen müssen.

\section{Augenverletzungen durch}

Feuerwerkskörper als globales Problem

Die SOE hat mit Unterstützung des ICO kürzlich die erste internationale Umfrage zu feuerwerksbedingten Augenverletzungen durchgeführt. Aus 25 Ländern wurden 425 Verletzte gemeldet, was verdeutlicht, dass es sich um ein globales Problem handelt, von dem in der vorliegenden Arbeit nur die Spitze des Eisbergs sichtbar wird. Die Mehrheit der Patienten waren Männer (90\%) und Kinder, und $50 \%$ waren Zuschauer; $12 \%$ der Unfälle endeten mit einem blinden Auge, und $5 \%$ der Augen mussten entfernt werden. Die weltweiten Daten sind mit denen der niederländischen Umfrage vergleichbar (- Tab. 4).

Ergebnisse einer deutschlandweiten Umfrage unter diensthabenden Augenkliniken, die von 2016 bis 2019 durchgeführt wurde, fanden bei rund 1400 Patienten eine deutlichen Überzahl von Männern (75\%) im jüngeren Alter $(60 \%$ waren 25 Jahre oder jünger) und Zuschauern oder Passanten
(60\%). Begleitende Verletzungen des zweiten Auges, des Gesichts und der Hände waren häufiger bei Kindern, die überwiegend durch Knallkörper verletzt wurden. Diese Daten sind vergleichbar mit den Ergebnissen aus den Niederlanden, wo die Vorschriften ähnlich sind, und zeigen deutlich die Notwendigkeit effektiverer Schutzmaßnahmen und intensiver Öffentlichkeitsarbeit (s. diese Ausgabe).

\section{》) Die Daten zeigen die Notwendigkeit und den Effekt ef- fektiver Schutzmaßnahmen und intensiver Öffentlichkeitsarbeit}

In Australien sind seit 1987 keine privaten Feuerwerke mehr erlaubt. Seither wurden keine feuerwerksbedingten Augenverletzungen beschrieben. Es gibt jedoch eine Ausnahme: In den Northern Territories (Hauptstadt: Darwin) wird der Territory Day (1. Juli) gefeiert, der die einzige Ausnahme vom totalen Verbot von privaten Feuerwerken darstellt. Die einzigen australischen Augenverletzungen durch Feuerwerk 2018 wurden daher in Darwin beschrieben. In der Republik Irland trat bereits 1875 ein Feuerwerksverbot in Kraft, hier können die Verbraucher heute Feuerwerkskörper der Kategorie F1 mit sehr geringer Gefährdung (z. B. Tischfeuerwerk, Weihnachtskracher und einige Wunderkerzen) erwerben. Professionelle Feuerwerksshows sind ebenfalls erlaubt. Die an das finnische Parlament 


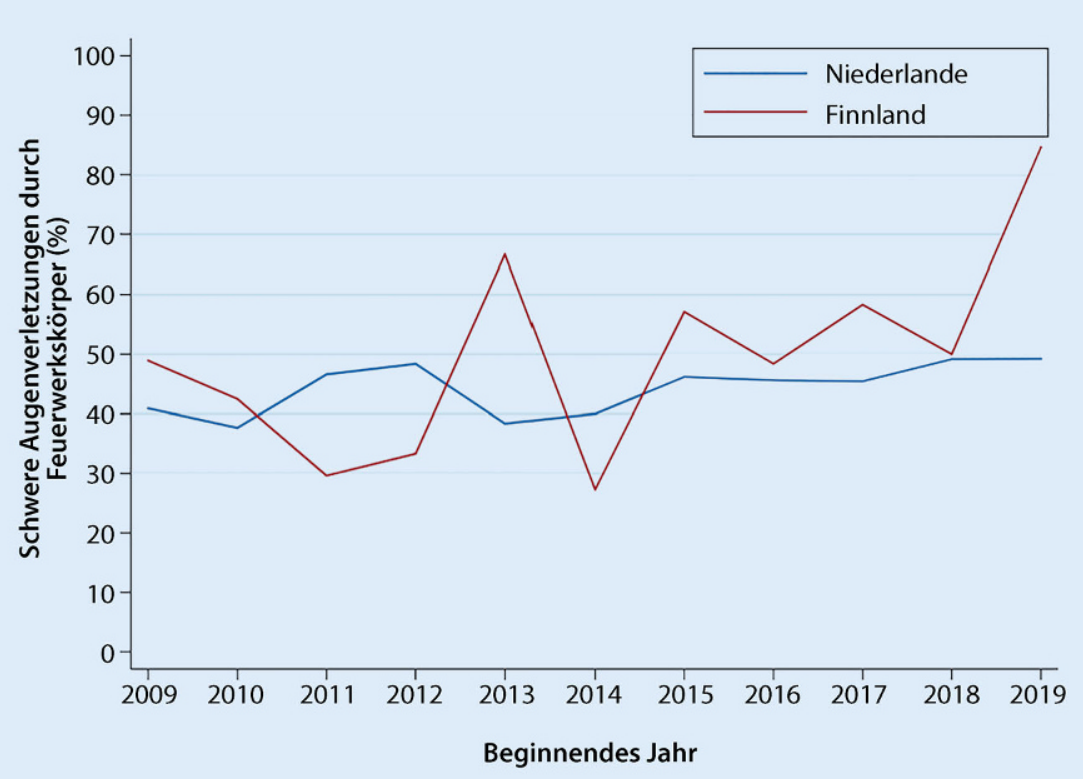

Abb. 5 A Anteil der schweren feuerwerksbedingten Augenverletzungen, die bleibende Schäden verursacht haben (Niederlande) und der mittleren und schweren Augenverletzungen, die Folgeschäden haben können (Finnland) zwischen 2008 und 2019. Die Methoden der Datenerhebung unterscheiden sich, sodass ein Vergleich der relativen Daten zwischen den Ländern nicht so zuverlässig ist wie ein Vergleich im longitudinalen Zeitverlauf für jedes Land

gerichtete Bürgerpetition hat sich eine vergleichbare Regelung zum Ziel gesetzt. Evidenz dazu legt die Studie von Wisse vor, die eine Reduktion der Feuerwerksbedingten Verletzungen um $87 \%$ durch ein Verbot von privatem Feuerwerk zeigt [7].

In den Vereinigten Staaten wurden 2017 etwa 1300 Augenverletzungen durch Feuerwerkskörper behandelt, 36 \% bei Kindern unter 15 Jahren, und $50 \%$ der Patienten waren jünger als 20 Jahre, so die Consumer Product Safety Commission (CPSC) [1]. Acht Betroffene starben nach Feuerwerksunfällen. Augenverletzungen machten $14 \%$ und Verbrennungen $53 \%$ der feuerwerksbedingten Verletzungen aus, die in Notfallambulanzen und -kliniken behandelt wurden. Knallkörper und Raketen verursachten mehr als die Hälfte der Verletzungen. Für die Datenerhebung wurde das National Electronic Injury Surveillance System (NEISS) verwendet. Aus diesen Daten geht hervor, dass zwischen 1999 und 2017 schätzungsweise 40 Personen pro 1 Mio. Einwohner Feuerwerksverletzungen erlitten haben. Männer machten dabei $70 \%$ aller Verletzten aus. Rund $50 \%$ dieser Augenverletzungen betrafen
Umstehende oder Passanten was vergleichbar ist mit den Ergebnissen der vorliegenden Studie.

\section{Was können Augenärzte} tun, um feuerwerksbedingte Augenverletzungen zu vermeiden?

Das öffentliche Bewusstsein für die Gefahr von Verletzungen durch Feuerwerkskörper muss geweckt werden. Dies erfordert eine verstärkte Aufklärung der Öffentlichkeit, insbesondere in der Zeit um die betroffenen Feiertage. Als Augenärzte müssen wir die Aufmerksamkeit der Medien nutzen, um die Öffentlichkeit zu warnen, und uns für Veränderungen der gesetzlichen Regelungen einsetzen. Mit dem Ziel, den privaten Konsum von Feuerwerkskörpern zu reduzieren, haben Augenärzte in den Niederlanden ein 10-Stufen-Programm entwickelt, um die Öffentlichkeit $\mathrm{zu}$ informieren und die Wahrnehmung des Problems durch die Gesetzgeber zu stärken [3].

Die 10 Schritte des gesellschaftlichen Aktionsplans für „Ärzte auf den Barrikaden" sind:

- Machen Sie eine Bestandsaufnahme des Problems und erklären Sie der
Öffentlichkeit, warum eine gesellschaftliche Veränderung notwendig ist.

- Machen Sie sich die Daten zu eigen und erklären Sie in einfachen Worten Ihre medizinische Expertise des Problems.

- Fassen Sie die Daten zusammen und veröffentlichen Sie sie.

- Schaffen Sie Verständnis und Unterstützung für Ihre lokalen, nationalen und internationalen Kollegen.

- Binden Sie die Medien ein (Social Media, TV, Radio, Printmedien).

- Schaffen Sie Unterstützung in der Bevölkerung.

- Erstellen Sie eine Community und starten Sie eine eigene Website.

- Seien Sie gerüstet für Gegenangriffe etwa wegen des kommerziellen Interesses der Feuerwerksindustrie.

- Bieten Sie eine sichere Alternative, wie z. B. öffentliches professionelles Feuerwerk.

- Erstellen Sie einen mittelfristigen und einen langfristigen Plan und bleiben Sie dran.

\section{Schlussfolgerung}

Augenverletzungen durch Feuerwerkskörper treten weltweit auf, am häufigsten dort, wo es keine oder nur unzureichende gesetzliche Regulierungen gibt. In Ländern, in denen die Verwendung von privaten Feuerwerken begrenzt ist, werden weniger Verletzungen registriert. Diese Regelungen zielen darauf ab, die zulässigen Zeiten für den Verkauf und die Verwendung von Feuerwerkskörpern zu begrenzen, das zulässige Alter für die Verwendung von Feuerwerkskörpern zu erhöhen und gezielte Verbote für bestimmte besonders verletzungsgefährdende Produkte zu erlassen. Außerdem sollte für den Gebrauch von Schutzbrillen geworben werden.

Feuerwerksbedingte Augenverletzungen sind ein internationales Problem, das eine globale Lösung erfordert $[5,6]$. Wo es offene Handelsgemeinschaften wie den EU-Binnenmarkt gibt, ist es schwierig, in einem Land Beschränkungen für den Verkauf bestimmter oder aller Arten von Feuerwerkskörpern zu schaffen. Der ungehinderte Waren- und Personenverkehr 


\begin{tabular}{|c|c|c|c|c|c|c|c|c|c|c|c|}
\hline Augenverletzungen & $\begin{array}{l}2008 / \\
2009\end{array}$ & $\begin{array}{l}2009 / \\
2010\end{array}$ & $\begin{array}{l}2010 / \\
2011\end{array}$ & $\begin{array}{l}2011 / \\
2012\end{array}$ & $\begin{array}{l}2012 / \\
2013\end{array}$ & $\begin{array}{l}2013 / \\
2014\end{array}$ & $\begin{array}{l}2014 / \\
2015\end{array}$ & $\begin{array}{l}2015 / \\
2016\end{array}$ & $\begin{array}{l}2016 / \\
2017\end{array}$ & $\begin{array}{l}2017 / \\
2018\end{array}$ & $\begin{array}{l}2018 / \\
2019\end{array}$ \\
\hline Patienten & 47 & 40 & 27 & 21 & 15 & 11 & 7 & 31 & 12 & 12 & 13 \\
\hline Aktiver Feuerwerker & 31 & $\mathrm{~N} / \mathrm{A}$ & 20 & 14 & 11 & 4 & 3 & 23 & 7 & 6 & 10 \\
\hline Umstehende & 16 & N/A & 7 & 7 & 4 & 7 & 4 & 8 & 5 & 6 & 3 \\
\hline Augen & 50 & N/A & $\mathrm{N} / \mathrm{A}$ & $\mathrm{N} / \mathrm{A}$ & 18 & 14 & 8 & 37 & 12 & 13 & 13 \\
\hline Milde Verletzung & 24 & 23 & $\mathrm{~N} / \mathrm{A}$ & 13 & 5 & 8 & 3 & 16 & 5 & 6 & 2 \\
\hline Mäßige Verletzung & 12 & 13 & $\mathrm{~N} / \mathrm{A}$ & 4 & 5 & 2 & 3 & 10 & 5 & 4 & 8 \\
\hline Schwere Verletzung & 11 & 4 & $\mathrm{~N} / \mathrm{A}$ & 4 & 5 & 1 & 1 & 5 & 2 & 2 & 3 \\
\hline
\end{tabular}

Tab. 4 SOE(European Society of Ophthalmology)-Umfrage 2017/18: $n=475$ Verletzte aus 25 Ländern

\begin{tabular}{|c|c|c|}
\hline Argentinien & Guatemala & Pakistan \\
\hline Australien & Ungarn & Philippinen \\
\hline Bangladesch & Indien & Russland \\
\hline Brasilien & Indonesien & Ruanda \\
\hline Kanada & Irland & Suriname \\
\hline Tschechien & Italien & Großbritannien \\
\hline China & Litauen & USA \\
\hline Curaçao & Mexico & \\
\hline Deutschland & Niederlande & \\
\hline \multicolumn{3}{|c|}{$\begin{array}{l}n=475 ; 50 \% \text { der Verletzten waren Umste- } \\
\text { hende oder Passanten (35\% erlitten einen } \\
\text { dauerhaften Schaden, } 12 \% \text { erblindeten } \\
\text { nach Gesetz, } 5 \% \text { Enukleationen, } 90 \% \text { Män- } \\
\text { ner, } 50 \% \text { Kinder oder Jugendliche) }\end{array}$} \\
\hline
\end{tabular}

soll gewährleistet bleiben. Daher befürworten und unterstützen die überregionalen ophthalmologischen Organisationen (SOE, ICO, IAPB und Vision 2020) die Bemühungen um ein weltweites Verbot von privat genutzten Feuerwerkskörpern.

\section{Fazit für die Praxis}

- Feuerwerksbedingte Augenverletzungen treten weltweit insbesondere bei privaten Feuerwerken auf.

- Rund $75 \%$ der Verletzten sind Jungen und Männer.

- Etwa die Hälfte der Verletzten sind Zuschauer oder Passanten.

- Schutzbrillen können Verletzungen verhindern und deren Schäden mindern.

- In Ländern, in denen die Verwendung von privaten Feuerwerken begrenzt ist, werden weniger Verletzungen registriert.

- Die ophthalmologischen Organisationen (DOG, SOE, ICO, IAPB und Vision 2020) befürworten und unterstützen die Bemühungen um ein weltweites Verbot von privat genutzten Feuerwerkskörpern.

\section{Korrespondenzadresse}



Jan Tjeerd de Faber

Augenklinik Rotterdam Rotterdam, Niederlande j.defaber@oogziekenhuis.nl

\section{Einhaltung ethischer Richtlinien}

Interessenkonflikt. J.T. de Faber, A. Gabel-Pfisterer und T.T. Kivelä geben an, dass kein Interessenkonflikt besteht.

Für diesen Beitrag wurden von den Autoren keine Studien an Menschen oder Tieren durchgeführt. Für die aufgeführten Studien gelten die jeweils dort angegebenen ethischen Richtlinien.

Open Access. Dieser Artikel wird unter der Creative Commons Namensnennung 4.0 International Lizenz (http://creativecommons.org/licenses/by/4.0/deed. de) veröffentlicht, welche die Nutzung, Vervielfältigung, Bearbeitung, Verbreitung und Wiedergabe in jeglichem Medium und Format erlaubt, sofern Sie den/die ursprünglichen Autor(en) und die Quelle ordnungsgemäß nennen, einen Linkzur Creative Commons Lizenz beifügen und angeben, ob Änderungen vorgenommen wurden.

\section{Literatur}

1. CPSC (2018) CPSC 2017 fireworks injuries annual report USA. https://www.cpsc.gov/ResearchStatistics/Fuel-Lighters-and-Fireworks1. Zugegriffen: Mai 2019
2. de Faber JT (2009) Fireworks injuries treated by Dutch ophthalmologists New Year 2008/09. Ned TijdschrGeneeskd 153:A507

3. de Faber JTHN, van Zuilen M, Snouck Hurgronje M, Keunen J (2017) Social awareness campaign for a ban on consumer fireworks: 10 tips; doctor on the barricade. Ned Tijdschr Geneeskd 161:D2249

4. Frimmel S, de Faber JT, Wubbels RJ (2018) Type, severity, management and outcome of ocular and adnexal firework-related injuries: the Rotterdam experience. Acta Ophthalmol 96(6):607-615

5. Toth G, Resnikoff S, de Faber JT, Nemeth J (2019) Preventing blindness and visual impairment in Europe: What do we have to do? Eur J Ophthalmol 29(2):129-132

6. Van Yperen DT et al (2018) Firework injuries in the south-western region of the Netherlands around the turn of the year. Ned Tijdschr Geneeskd 29(162):2017-2018

7. Wisse RP, Bijlsma WR, Stilma JS (2010) Ocular firework trauma: a systemic review on incidence, severity, outcome and prevention. Brit J Ophthalmol 94(12):1586-1591 